\title{
Ética e investigación en la historia de la malaria
}

\author{
Walter Ledermann D. y Guido Valle B.
}

\section{Hospital Luis Calvo Mackenna, Santiago de Chile. Laboratorio de Microbiología (WLD) Departamento de Medicina Nuclear, Instituto Científico Hospital Casa Sollievo della Sofferenza, San Giovanni Rotondo, Italia (GV) \\ Recibido: 11 de agosto de 2009 Aceptado: 28 de agosto de 2009 \\ Correspondencia a: Walter Ledermann D. oncemayor@gmail.com \\ Guido Valle prof.gvalle@gmail.com}

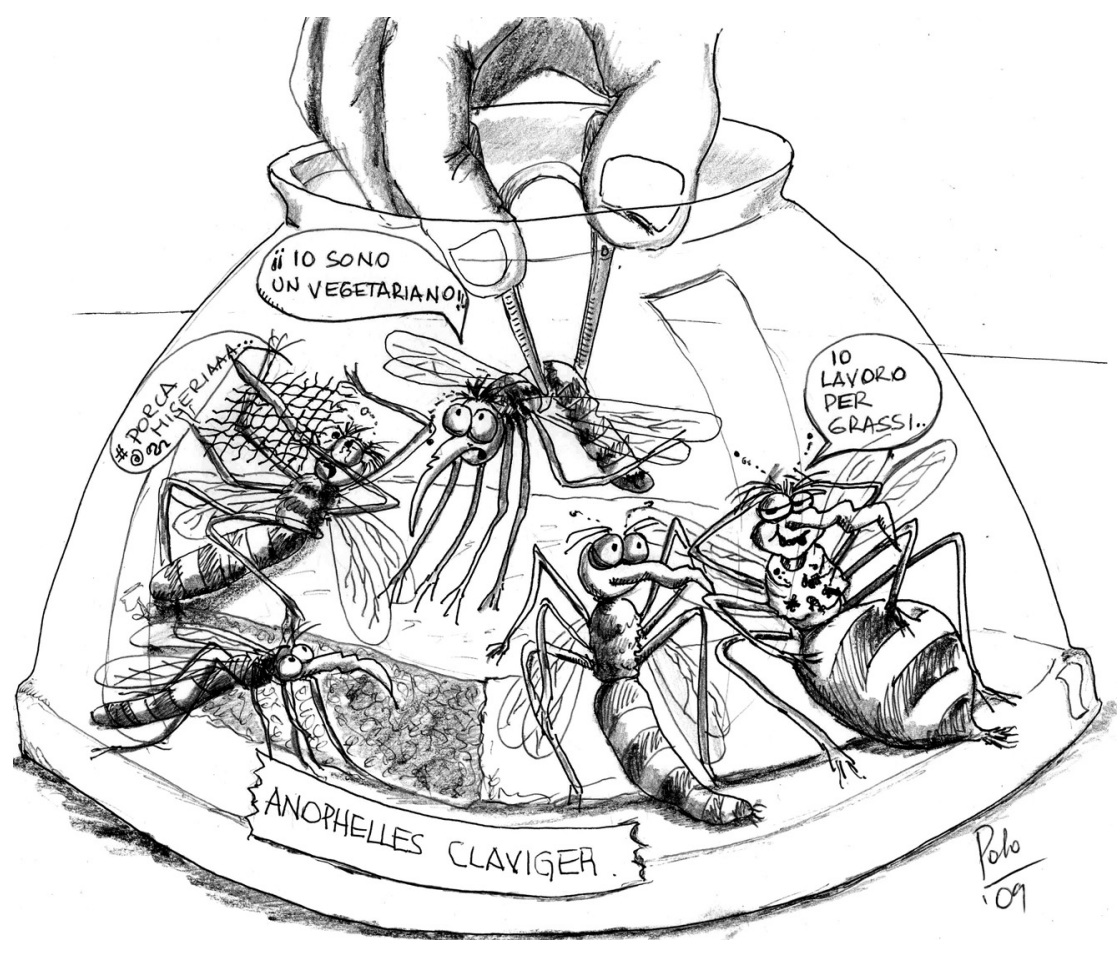
clinical trials with antimalarial drugs.

\section{Introducción}

os descubrimientos de Pasteur y Koch hacia el fin del siglo XIX despertaron el interés por la microbiología, impulsando el desarrollo de la investigación en el campo de las enfermedades infecciosas. En Italia, donde la malaria era endémica y gravísimo problema de salud, una pléyade de investigadores compitió por descubrir tanto el agente causal como los mecanismos de transmisión y, al

\section{Ethics and research in the history of malaria}

Clinical research is absolutely necessary for the development of medical knowledge. However, progress of Medicine has not always been obtained respecting human rights. From 1880 onwards, after Laveran's discovery of the protozoan nature of malaria, the increase in knowledge on paludism has been remarkable. Much of the knowledge gained up to the middle of the twentieth century was based although on highly questionable experiments according to modern ethical standards in the fields of biology and phisiopathology, and specially in therapeutic

Key words: Malaria, paludism, ethics, bioethics, history.

Palabras clave: Malaria, paludismo, ética, bioética, historia. a causal, en tanto que el segundo, liderado por el zoólogo Battista Grassi, intentaba descubrir el vector que transportaba esta sangre del enfermo al susceptible. Siendo sus objetivos distintos, pero complementarios, ambos inocularon humanos con sangre que contenía el parásito causal.

\section{Experiencias de Marchiafava}

Las experiencias de Marchiafava se realizaron inoculando en individuos sin malaria sangre obtenida de enfermos en su período febril. En esta línea, se ha sostenido que el pionero en la materia fue el ruso Alexander Samoilovitch Rosenblium (1826-1902), pero éste no tenía interés en la malaria, siendo sólo el primero en tratar la neurosífilis mediante la inoculación de material infeccioso, utilizando sangre de enfermos con fiebre recurrente y también de otros enfermos "que habrían contraído malaria o tifoidea": nótese que no afirma que sufrieran, verdaderamente, de malaria. Según Rosenblium, entre 1876 y 1877 habría tratado a 32 pacientes psicóticos con este método, provocando fiebre en 32, de los cuales 21 fueron curados, tres mejoraron y ocho no tuvieron cambio alguno ${ }^{1}$. 
El pionero en la inoculación con sangre de enfermos maláricos fue el alemán Karl Gerhardt (1833-1902), quien logró inducir malaria en dos voluntarios, controlando luego los accesos febriles con quinina. Gerhardt pensaba que esta enfermedad se debería a "un veneno que se multiplicaba dentro del cuerpo humano y era transportado por un organismo inferior". A su juicio, si las experiencias anteriores sólo habían producido resultados "equívocos", era porque no se habían respetado cuatro condiciones básicas:

- El experimento debía realizarse en una región libre de malaria.

- El paciente que proporcionaba la sangre no debía tener ninguna otra enfermedad infecciosa.

- Los sujetos a inocular debían ser voluntarios y el experimento no podría obligarlos a sacrificar una oportunidad para desempeñarse en un empleo lucrativo.

- Las curvas de temperatura de los sujetos debían registrarse desde mucho antes de la inoculación, para asegurarse que estaban libres de fiebre ${ }^{2}$.

Desde el punto de vista ético merece la pena destacar el contenido del tercer punto, donde se establece, aparte del carácter voluntario de los sujetos, que su participación "no debe obligarlos a sacrificar una oportunidad para desempeñarse en un empleo lucrativo". Aunque pareciera preocuparse más del lucro cesante que de la salud de los voluntarios, constituye una consideración muy avanzada para la época- y muy práctica.

En 1881 Marchiafava había inoculado perros con sangre de enfermos maláricos, como antes había hecho Cuboni, mientras que Dochmann y Leoni emplearon como fuente infectante el contenido de herpes labial de maláricos, para concluir con este exitoso intento de Carl Gerhardt. Con estos antecedentes, el investigador italiano, en compañía de Angelo Celli y Umberto Mariotti quienes ya tenían experiencia con el método, inició su investigación en el Hospital del Santo Espíritu (Ospedale Santo Spirito), cerca del Vaticano, en Roma, publicándola en $1885^{3}$. Este hospital, instituido por el Papa Inocencio III en 1201, el primer hospital propiamente tal en Europa ${ }^{4}$, reunía las mejores condiciones para la investigación de la malaria, por el gran número de enfermos con esta patología que atendía, bastando señalar que en cuatro años, entre 1892 y 1895 , ellos llegaron a 20.855 , en tanto que el San Giovanni reunió 2.603, cifras que pueden dar una idea de la magnitud del problema en Italia: no por nada el nombre malaria -mal aire- nació en la península ${ }^{5}$.

La investigación comprendió cinco pacientes con enfermedad nerviosa crónica, quienes se prestaron voluntariamente a la experiencia. Se consideraron circunstancias favorables para asegurar la ausencia de una infección malárica de otro origen durante el estudio: que el hospital estaba situado en una zona de Roma libre de esta enfermedad; que los voluntarios no tenían antecedentes de infección anterior, salvo uno, quien la había padecido muchos antes, trabajando luego por años en una zona endémica sin presentar nuevos accesos febriles; que ninguno había tenido fiebre en los últimos 15 a 20 días, constatado con rigurosas mediciones diarias.

No se menciona ni el número ni la edad de los pacientes que sirvieron como fuente de infección, cuya sangre fue utilizada en algunos casos en distintas ocasiones para inocular a diferentes receptores. El volumen extraído nunca superó un gramo, siendo de inmediato inoculado al receptor, ya sea en forma intravenosa o subcutánea. El sujeto que sufrió más inoculaciones, P.D., recibió dos con 0,5 g vía subcutánea y luego 0,5 y 1 g vía intravenosa, en tanto que B.A. sólo recibió $1 \mathrm{~g}$ intravenoso por una sola vez.

Las inoculaciones de los tres primeros voluntarios - PD, LS y ME- reprodujeron los accesos febriles propios de la enfermedad, con una duración de cuatro a cinco días, acompañados de las características alteraciones sanguíneas y con respuesta favorable al uso de quinina. De aquí se desprende la primera conclusión de Marchiafava y Celli: La inyección de poca cantidad de sangre de enfermos maláricos febriles puede reproducir una verdadera y propia infección malárica, que se caracteriza por especiales alteraciones de los glóbulos rojos.

En cambio, los voluntarios cuarto y quinto -DGS y BA- sólo presentaron fiebre esporádica o irregular, independiente de la administración de quinina. De estos dos fracasos, Marchiafava y Celli deducen una segunda conclusión: La inyección de sangre de maláricos febriles no siempre reproduce la fiebre malárica.

Las conclusiones son lógicas e impecables para la época, pero la muestra es pequeña y falta la prueba principal: el aislamiento en la sangre del donante y del receptor del agente etiológico de la enfermedad, por entonces aun no cultivado y todavía en discusión. Y faltaba también saber cómo llegaba a la sangre este desconocido parásito.

\section{Experiencias de Grassi}

La hipótesis culpando al mosquito comúnmente llamado zanzara no era nueva en Italia, remontándose hasta un muy citado párrafo de Columella, en el siglo I D.C 6 , pasando por Laveran y otros, hasta llegar a su completa formulación con Bignami, quien opinaba que el agente malárico no se transmitía ni por el agua ni por al aire, tomándolo el mosquito del suelo. Por otra parte, el descubrimiento por Ronald Ross del mosquito Culex como vector de la malaria aviar, que le valdría el Premio Nobel en 1902, dio un espaldarazo decisivo a la teoría ${ }^{7}$.

Grassi pensó en un problema de especificidad, es decir, que había un mosquito específico para la malaria, y viajó a través de todo el país analizando los mosquitos de las zonas endémicas y no endémicas, hasta concluir que el 
Anopheles claviger, más grande que los Culex y por ello llamado zanzarone, era el insecto sospechoso. Entonces, como era uno zoologo y no un clínico, recurrió a los doctores Giuseppe Bastianelli y al ya mencionado Bignami, para demostrar su tesis con enfermos del Hospital del Santo Espíritu.

En los primeros tiempos en que me ocupaba de la malaria-declara Grassi-creí necesario hacer una prueba en el hombre, pero no supe vencer la repugnancia que me inspiraba cualquier prueba eventualmente dañina sobre una persona, la cual, pese a ser informada previamente, no puede tener un conocimiento cabal del peligro a que se expone. Quizás por ello decidió hacer la primera experiencia en su propia persona y soltó varios Anopheles claviger en su dormitorio, sin que ninguno de ellos se dignara a picarlo; pese a las precauciones tomadas, uno escapó de la habitación y fue a picar a su madre, afortunadamente sin consecuencias.

Entonces, olvidando sus prevenciones, soltó sucesivamente varios Anopheles sobre dos enfermos del Hospital del Santo Espíritu. El segundo era un viejo que no había tenido malaria y llevaba seis años hospitalizado en la sección del hospital a cargo de Bastianelli: "el perfil del sujeto de nuestro experimento no podía ser mejor, como tan bien lo ha dicho Bignami”, expresa Grassi, relatando como durante un mes, noche a noche, soltaron sobre el infeliz paciente hasta un centenar de mosquitos, encontrándose en la mañana "varios Anopheles con el abdomen lleno de sangre". Sus sufrimientos cesaron cuando finalmente tuvo fiebre, luego de una noche en que soltaron muy pocos Anopheles, más o menos unos diez. En su defensa, dice el investigador: "la infección fue inmediatamente curada y vencida con una inyección de quinina". Esta resolución de la fiebre con la quinina es una ulterior prueba de la naturaleza malárica de los accesos febriles (criterio "ex adiuvantibus").

En las experiencias previas, tendientes a demostrar qué especies del género Anopheles transmitían el parásito, se utilizaron mosquitos que ya eran portadores al momento de ser capturados, pero la necesidad obligó en ocasiones a obtener el plasmodio haciendo que los insectos picaran a enfermos: a uno de ellos, con la sangre rica en parásitos, lo hicieron picar por cinco insectos en media hora. Ya antes Ronald Ross había hecho picar a un enfermo una decena de veces: era, pues, un método habitual en la investigación de la época.

¡Éxito total! "Con Bastianelli y Bignami hemos demostrado que el Anopheles claviger es apto para propagar la malaria humana; los experimentos fueron realizados y repetido cien veces por alguno de nosotros". Sin embargo, para mayor comprobación, Bignami y Bastianelli continuaron los terribles experimentos, el segundo sobre "N.N., un joven robusto, que no ha tenido fiebre y se encuentra en el hospital cerca de tres años...” El tercero tuvo, "en total", apenas cinco picaduras; posteriormente hubo uno que recibió 40 picaduras en un solo día...8.

Todos los pacientes dieron su "consentimiento informado", pero el mismo investigador reconoce desde un principio que los sujetos no podían comprender en su totalidad el riesgo al que se exponían. La Declaración de Helsinki, que regula la investigación médica en humanos, muy claramente expresa en su punto 23 , refiriéndose a la obtención del conocimiento informado: "el médico debe tener especial cuidado cuando el individuo está vinculado por él por una relación de dependencia o si consiente bajo presión”. ¿Qué mayor relación de dependencia que la del primer paciente, un anciano que llevaba seis años al cuidado de Bastianelli ? "No podía ser mejor", dicen Grassi y Bignami, los otros dos investigadores, comentando la situación del paciente.

El mismo artículo 23 continúa: "En un caso así, el consentimiento informado debe ser obtenido por un médico bien informado, que no participe en la investigación y que nada tenga que ver con aquella relación". Esta posibilidad con seguridad no pasó por la mente de los investigadores, siendo inconcebible en esa época. Tampoco evaluaron si la persona era incapaz, o inhábil física o mentalmente de otorgar consentimiento, como se expresa en el artículo 24, en cuyo caso se requiere el consentimiento de un tutor. Los enfermos de Marchiafava sufrían de patología neurológica y un individuo afectado de mielitis transversa, enteramente dependiente del personal del hospital, está claramente en una situación de extrema indefensión para atreverse a contrariar la voluntad de su médico?.

Podemos decir, en descargo de estos audaces investigadores, que sus objetivos eran nobles y de acorde con varios artículos de la mencionada Declaración en el sentido que "la importancia del objetivo es (era) mayor que el riesgo" ...y de los posibles beneficios para la humanidad, derivados del mejor conocimiento de la enfermedad, con las posibilidades terapéuticas y epidemiológicas que de éste deriven. Y también podemos decir en su descargo que, muy recientemente, estas experiencias con voluntarios sometidos a picaduras de mosquitos infectados se han efectuado repetidas veces, rematando en este siglo con un trabajo destinado a evaluar la seguridad de los protocolos actuales y conocer las tasas de infección, objetivos que desmerecen junto a los de Marchiafava y Grassi ${ }^{10}$.

Volvamos por un momento a Grassi, quien se lanza en busca de una "contraprueba", consistente en demostrar que "evitando la picadura del Anopheles se evita de manera absoluta la fiebre”. Ampliando experimentos anteriores ingleses e italianos, que le parecieron de escasa envergadura, en el 1900 eligió una zona de la provincia de Salerno, la llanura de Capaccio, de altísima endemia, para realizar una prueba amplia en un grupo de trabajadores ferroviarios. Durante la estación "malárica", del 26 de junio al 14 de octubre, protegió con una fina 
rejilla metálica las habitaciones de los voluntarios, de manera que los mosquitos no pudieran de noche entrar a picarlos. Éstos fueron en total 112, incluyendo familiares de los trabajadores, tanto así que 32 eran niños menores de 11 años. Un grupo de 415 individuos sin rejillas en las ventanas sirvió como control. Los resultados fueron concluyentes: en el grupo protegido hubo cinco casos de fiebre, que correspondieron a recidivas, sin que se presentara ninguna infección primaria, en tanto que enfermó la totalidad de los 415 no protegidos que sirvieron de grupo control, incluyendo los 91 voluntarios que ocuparon las caseta ferroviarias ${ }^{8}$.

Desde el punto de vista ético, podríamos objetar la mantención de un grupo control a medida que el avance de la estación malárica iba mostrando la tremenda ventaja de la rejilla de protección. Comparemos la situación con la producida casi un siglo después, con el famoso protocolo 076 para estudiar la profilaxis durante el embarazo contra la infección por VIH en el recién nacido: cuando, en pleno desarrollo, los beneficios se demostraron aplastantes, los investigadores extendieron la profilaxis al grupo control ${ }^{11}$.

\section{Ensayos de fármacos antimaláricos}

Definidos ya claramente el vector y el mecanismo de transmisión de la enfermedad, la investigación se centró tanto en su fisiopatología como en la terapéutica. Así, las sucesivas experiencias del italiano Camillo Golgi, Premio Nobel de Medicina 1906 por sus investigaciones en neurohistología y por la descripción del aparato intracelular que hoy lleva su nombre, demostraron que el cuadro clínico de la malaria podía ser provocado por diferentes tipos de Plasmodium, y que las crisis febriles correspondían a la rotura de los eritrocitos y a la liberación de los merozoítos.

Al inicio de la I Guerra Mundial los aspectos esenciales de la fisiopatología clínica de la malaria eran ya suficientemente conocidos, mientras que desde el punto de vista terapéutico la quinina, obtenida mediante extracción, representaba por más o menos dos siglos y medio el único principio activo. Sin embargo, durante el conflicto los dos bandos enfrentados serían golpeados por la malaria en diversos teatros de operaciones, por cuanto la situación bélica haría difícil el aprovisionamiento del medicamento.

En Alemania, el progreso de la industria química de los colorantes permitió llevar a cabo, en los años sucesivos a esta guerra, un programa de investigación sistemática para lograr la síntesis de moléculas activas contra los protozoos en general y contra el plasmodio malárico en particular. Estas investigaciones se llevaban a cabo en el laboratorio Bayer de Elberfeld a partir de colorantes como el azul de metileno y el tripan rojo, que se habían revelado útiles para colorear el plasmodio de acuerdo al principio, enunciado por Paul Ehrlich, para quien el hecho que una sustancia colorease selectivamente un microorganismo demostraba la presencia de un tropismo de la molécula colorante hacia él. Este tropismo se debía a la existencia en el microorganismo de un grupo químico que llamó receptor (introduciendo este afortunadísimo término al vocabulario médico-biológico) del colorante. Tal molécula colorante y sus derivados eran entonces potenciales "proyectiles mágicos", capaces de golpear selectivamente al microorganismo.

En realidad, ya hacia 1891 Paul Ehrlich y Paul Guttman habían ensayado la posibilidad del uso clínico del azul de metileno sobre dos pacientes afectados de malaria, pero este compuesto se había mostrado menos eficaz que la quinina, siendo por lo tanto abandonado ${ }^{12}$. A partir de este colorante se sintetizó en 1926 la plasmoquina que, además de ser el primer antimalárico sintético, era activo contra los hipnozoitos y los gametocitos. La misma industria, en al ámbito del mismo programa de investigación, desarrolla en 1932 el colorante acridínico atebrina ${ }^{13}$.

El interés suscitado por tales estudios no se había apagado y encontró amplio desarrollo en el período comprendido entre las dos guerras mundiales, sobre todo en Alemania. Pero todos estos fármacos necesitaban un adecuado ensayo clínico, porque los resultados obtenidos en el laboratorio farmacéutico empleando el modelo de la malaria aviar no siempre correspondían a los registrados sobre el hombre, y porque los canarios empleados en esta experimentación estaban infectados con el Plasmodium relictum. Y una vez más, como en los tiempos de Celli y Marchiafava, los enfermos mentales fueron usados como cobayos, como ocurrió con la experimentación conducida por Franz Sioli, director del Hospital Psiquiátrico de Dusseldorf. También en Italia, en los años transcurridos entre las dos guerras mundiales, los ensayos de los fármacos antimaláricos y sus protocolos terapéuticos se efectuaban sobre este tipo de pacientes ${ }^{14}$.

El estallido de la II Guerra Mundial indujo en los dos bandos enfrentados una febril actividad de investigación, para poner a punto fármacos y protocolos terapéuticos idóneos para proteger a las tropas de la malaria y de las enfermedades infecciosas en general.

En E.U.A. el Committee for Medical Research inició evaluaciones de carácter farmacológico sobre pacientes sifilíticos sometidos a malariaterapia en el hospital Saint Elizabeth, de Washington. Sin embargo, como el número de sujetos luéticos no era absolutamente suficiente para la finalidad de esta investigación gubernamental, el doctor $\mathrm{G}$. Robert Coatney pidió y obtuvo autorización para efectuar el ensayo de los nuevos fármacos antimaláricos sobre los reclusos de la penitenciaría federal de Atlanta, quienes habrían voluntariamente aceptado someterse al experimento, aunque resulta difícil comprender cuán libre podía ser el consentimiento de un prisionero. Un ala entera de la prisión fue dedicada a laboratorio, siendo interesante notar que de tal estructura científico-carcelaria partió el camino 
que luego llevaría a los actuales y famosos Centers for Disease Control de Atlanta, de relevancia mundial en la prevención y el control de las enfermedades infecciosas ${ }^{15}$.

\section{Experiencias de Schilling}

En Alemania, los estudios fueron encabezados por Claus Schilling, quien había estudiado en Berlín en la escuela de Robert Koch. Nacido en Mónaco de Baviera el 5 de julio de 1871, se encontraba ya retirado en febrero de 1942, cuando recibió órdenes de Himmler para iniciar experimentos sobre prisioneros en el campo de concentración de Dachau. En breve síntesis, después de haber inducido la enfermedad en los prisioneros mediante picadura de anofeles infectantes o mediante inyecciones de esporozoítos, aplicaba diferentes métodos de tratamiento con quinina, pirifer, neosalvarsan, antipirina, piramidón y un compuesto denominado Behring 2516, pero sus métodos no respetaban los más elementales derechos humanos, por lo cual fue arrestado al término de la contienda y procesado en Nuremberg ${ }^{16}$.

Entre los testigos de la fiscalía estaba el doctor Franz Blaha, un médico checoeslovaco que fuera obligado a efectuar la autopsia sobre los prisioneros muertos a continuación de los experimentos de Schilling. Blaha declaró que entre treinta y cuarenta prisioneros murieron por la malaria, en tanto que un número comprendido entre trescientos y cuatrocientos fallecieron por enfermedades que resultaron fatales a causa del deterioro de la condición física provocado por la malaria. De este testimonio se desprendió, además, que muchas víctimas habían fallecido por sobredosis de neosalvarsan o de piramidón. Por tales razones Claus Schilling fue condenado a muerte y ahorcado $^{17,18}$.

En el curso de este artículo nos habíamos referido a las múltiples investigaciones que, si bien no recomendables desde el punto de vista ético, sobre todo en cuanto a la ausencia de un consentimiento informado válido, habían aportado en su mayor parte resultados de extrema importancia para la comprensión de la fisiopatología de la infección malárica, traduciéndose en conocimiento esencial para la lucha contra la enfermedad.

Algunos de los investigadores cuyo comportamiento sería hoy objeto de procedimiento penal, como Celli, Marchiafava y Grassi, obtuvieron importante reconocimiento por su actividad y después de su muerte se les han dedicado hospitales y escuelas. En algunos casos -Marchiafava y Grassi- han obtenido la designación de senadores del Reino de Italia. En cambio, Claus Schilling fue condenado a muerte y ajusticiado por sus experimentos.

Tal disparidad de criterio y de trato requiere, a nuestro juicio, algunas reflexiones. Desde un punto de vista estrictamente ético todos estos investigadores han violado, en sustancia, el juramento de Hipócrates en el punto en que se afirma: "Resguardaré la conservación de la vida por el bien del enfermo según mi fuerza y mi juicio, absteniéndome de producir daño u ofensa. No suministraré a nadie, ni aun cuando lo solicitase, fármaco mortal ni sugeriré semejante consejo" 19.

Aun así, no parece del todo recomendable medir con el metro de hoy comportamientos del pasado y así validarlos en base a criterios éticos fatigosamente establecidos después de muchos decenios.

De hecho, hasta fines de la II Guerra Mundial, la atención en el resguardo de los problemas de la bioética era extremadamente escasa y no existían, al menos en Italia, criterios o normativas específicas a los cuales debieran atenerse los investigadores. Por otra parte, las dimensiones y el peso social del "problema malaria" eran inmensos, comparables a los de una verdadera guerra, y estas evaluaciones de tipo "bélico" justificaron el empleo del contingente militar de la Cruz Roja Italiana en la lucha contra la malaria. Ello puede al menos justificar comportamientos como los de Celli, Marchiafava, Grassi y Gerhardt, cuyas investigaciones, por otra parte fundamentales, no se asociaron a un aumento seguro de la mortalidad en la población estudiada y se efectuaron sobre grupos muy limitados de personas. Frente a los miles de muertos que se registraban anualmente por la malaria sólo en Italia, exponer a sufrimiento y riesgo a un grupo restringido de personas, como sucede en la guerra, puede indudablemente constituir una atenuante muy fuerte para una actividad de investigación bastante desprejuiciada desde el punto de vista de la ética de los experimentos.

Por otra parte, la aceptación del riesgo, aun mortal, está ligado a la mayor parte de la actividad humana, como la conducción de automóviles, navegación marítima o aérea, actividad industrial, etc, y en la programación de la investigación científica aparece actualmente ético efectuar un análisis riguroso de la relación riesgo/beneficio. Por ejemplo, si trasladamos a la infectología el mismo criterio de evaluación, de uso corriente y jurídicamente validado para la investigación clínica con radiación ionizante sobre voluntarios, para el cual es aceptable correr un riesgo de muerte de 1: 1.000 para obtener un beneficio sustancial para la sociedad; de 1: 10.000 a 1: 100.000 para obtener un beneficio social de intermedio a moderado; y de 1: 1.000.000 para obtener un beneficio social limitado. En todos los casos se va efectuando un constante análisis estadístico de los resultados, a fin de concluir la investigación apenas se obtengan resultados estadísticamente significativos, limitando así al mínimo el número de personas expuestas al riesgo ${ }^{20,21}$.

Estos criterios y estas evaluaciones recién fueron formuladas en la segunda mitad del siglo XX, pero antes evidentemente la consideración del riesgo que podía soportar la persona objeto de la investigación se hacía "al ojo" de los investigadores. 
Muy diverso es el caso de Schilling en el campo de concentración de Dachau. Por cuanto la necesidad de obtener un instrumento de protección contra la malaria era esencial por motivos militares, se hace evidente que los experimentos se llevaron a cabo sin ningún cuidado por la vida de los prisioneros. No quisiéramos extendernos sobre los motivos que pueden haber inducido a Schilling a un comportamiento tan reprobable. Durante el proceso a cuyo término fue condenado a muerte, sostuvo en su defensa haber actuado por el bien de la humanidad y por necesidades de la guerra. Probablemente, a nuestro parecer, a la edad de más de setenta años, después de haber dedicado la vida al estudio de la malaria, quería dejar su nombre ligado a algún descubrimiento importante. Pero ello evidentemente no lo justifica ni mucho menos justifica los golpes y el maltrato que personalmente infería a los prisioneros, más allá de cualquiera experimentación científica.

Ciertamente los experimentos conducidos sin escrúpulos por Claus Schilling, sumados a los de otros "médicos" nazis tristemente célebres, fueron la base para la redacción de la "Declaración de Nuremberg", uno de los documentos más importantes de los cuales se originó la "Declaración de Helsinki", que constituye el primer documento internacional para la reglamentación de la investigación científica en el hombre que hoy rige, como propuesta fundamental, el válido consentimiento informado de la persona expuesta a la investigación.

\section{Resumen}

La investigación científica en clínica es absolutamente necesaria para el desarrollo del saber médico. No obstante, no siempre el gran progreso alcanzado por la Medicina se ha obtenido respetando la persona humana. En particular, a partir de 1880, cuando Laveran constató por primera vez la naturaleza protozoaria de la malaria, el desarrollo del conocimiento sobre el paludismo ha sido muy notable, pero hasta la mitad del siglo XX estos logros fueron, en muchos casos, fruto de experimentos que hoy resultan en extremo discutibles desde el punto de vista ético, tanto los dedicados al estudio de la biología y de la fisiopatología, como especialmente los ensayos de carácter farmacológico efectuados en individuos sanos para probar moléculas con acción antimalárica real o presunta.

\section{Referencias}

1.- Zakon S J. Alexander Samoilovitch Rosenblium. His contribution to fever therapy. Arch Derm Syph 1943; 48: 52-9.

2.- Codell Carter, K. The rise of causal concepts of disease: case histories. Ashgate Publishing Ltd, UK 2003; 165-6.

3.- Marchiafava E, Celli A. Nuove ricerche sulla infezione malarica. Annali di Agricoltura 1885; 96: 1-33.
4.- Massani, M. Le molte vite dell'Ospedale di S. Spirito dalle sue origini ai nostri giorni. Giornale di Medizina Militare 1983; 103 (1): 54-66.

5.- Snowden F M. La conquista della malaria. Una modernizzazione italiana 1900-1962. Giulio Einaudi editore, Torino 2000; 46 .

6.- Columella. Los doce libros de agricultura que escribiera en latín Lucio Junio Moderato Columela, traducidos al castellano por D. Juan María Álvarez de Sotomayor y Rubio. Imprenta de D. Miguel de Burgos, Madrid 1824; I (5): 22.

7.- Fantini B. Uno zoologo e la malaria : Battista Grassi e la scoperta del ciclo di trasmissione della malaria. En: Grassi B. Studi di uno zoologo sulla malaria. Giunti Gruppo Editoriale, Firenze 1998; 22.

8.- Grassi B. Studi di uno zoologo sulla malaria. Biblioteca della scienza italiana. Giunti Gruppo Editoriale, Firenze 1998; 89-276.

9.- Consejo de Organizaciones Internacionales de las Ciencias Médicas (CIOMS). Pautas éticas internacionales para la investigación biomédica en seres humanos. Ginebra 2002.

10.- Verhage D F, Telgt D S C, Bousema J T, Hermsen C C, van Gemert G J A, van der Meer J W M, et al. Clinical outcome of experimental human malaria induced by Plasmodium falciparum infected mosquitoes. Nth J Med 2005; 63: 52-8 .

11.- Connor E M, Sperling R S, Gelber R, et al. Reduction of maternal-infant transmission of HIV type 1 with zidovudine treatment. N Engl J Med 1994; 331: 1173-80.

12.- Guttman P, Ehrlich P. Ueber die Wirkung des Methylenblau bei Malaria. Berliner Klinische Wochenschrift 1891; 28: 953-6.

13.- Schlitzer M. Malaria chemotherapeutics Part I: History of antimalarial drug development, currently used therapeutics, and drugs in clinical development. Chem Med Chem 2007; 2: 944-989.

14.- Greenwood D. Historical perspective. Conflict of interest: the genesis of synthetic antimalarial agents in peace and war. J Antimicrob Chemother 1995; 36: 857-72.

15.- Sweeney A W. Wartime research on malaria chemotherapy. Parassitologia 2000; 42: 33-45.

16.- Euckart W U, Vondra H. Malaria and World War II: German malaria experiments 1939-45. Parassitologia 2000; 42: $53-8$.

17.- The trial of Prof. Dr. Klaus Schilling at Dachau. http://www. scrapbookpages.com/dachauscrapbook/Dachau Trials/Klaus Schilling.html.

18.- Economy-point.org. Claus's Schilling. http://www.economypoint.org/c/clauss-schilling.html

19.- Millikin D. Hippocrates, Oath of. En: Collier's Encyclopedia. Crowell Collier and MacMillan,Inc, USA 1967; 138.

20.- Radiological Protection in Biomedical Research - ICRP publication 62. Pergamon Press, New York, 1993.

21.- Radiation Protection 99. Guidance on medical exposures in medical and biomedical research. European Communities, 1998. 